\title{
Electromagnetic scattering of high-permittivity particles on a substrate
}

\author{
Philippe Gay-Balmaz and Olivier J. F. Martin
}

\begin{abstract}
We contribute to the study of the optical properties of high-permittivity nanostructures deposited on surfaces. We present what we believe is a new computational technique derived from the coupled-dipole approximation (CDA), which can accommodate high-permittivity scatterers. The discretized CDA equations are reformulated by use of the sampling theory to overcome different sources of inaccuracy that arise for high-permittivity scatterers. We first give the nonretarded filtered surface Green's tensor used in the new scheme. We then assess the accuracy of the technique by comparing it with the standard CDA approach and show that it can accurately handle scatterers with a large permittivity. (c) 2001 Optical Society of America
\end{abstract}

OCIS codes: $\quad 290.5880,240.0240,290.5850,260.2110$.

\section{Introduction}

The Green's tensor technique, also known as coupleddipole approximation (CDA) or discrete-dipole approximation, is a powerful tool to solve scattering problems. It has been applied to numerous configurations, ranging from scattering by interstellar graphite dust particles, ${ }^{1}$ scattering by irregular inhomogeneous particles, ${ }^{2}$ the optical properties of metal spheres in suspension, ${ }^{3}$ or the study of near-field optical microscopy ${ }^{4}$ and optical nanolithography. ${ }^{5}$

An attractive feature of the CDA is that it is also well suited for calculation of scatterers located above surfaces or embedded inside a multilayer background.6,7 In such cases the free-space Green's tensor is replaced with that corresponding to the surface or to the multilayer background. In this approach only the scatterers must be discretized, the background being taken into account in the Green's tensor.

Unfortunately, the CDA becomes less accurate when scatterers with a high permittivity are at hand. Refining the discretization mesh in that case does not necessarily increase the result accuracy, as was investigated in detail in Ref. 8. To overcome these limita-

The authors are with the Electromagnetic Fields and Microwave Electronics Laboratory, Swiss Federal Institute of Technology, ETH-Zentrum, ETZ, CH-8092 Zurich, Switzerland. P. GayBalmaz's e-mail address is balmaz@ifh.ee.ethz.ch.

Received 18 January 2001; revised manuscript received 7 May 2001.

0003-6935/01/254562-08\$15.00/0

(C) 2001 Optical Society of America tions, we introduced a modified CDA scheme based on sampling theory and using the so-called filtered Green's tensor. ${ }^{9}$ This scheme was shown to handle scatterers accurately with a permittivity as large as 10 , located in an infinite homogeneous background.

In the present paper we propose to extend this theory to small scatterers embedded inside or deposited on a surface. For this purpose, in Section 2, we derive the filtered Green's tensor associated with a surface. In Section 3 we assess the accuracy of this new scheme by comparing results obtained with the filtered and the nonfiltered schemes for scatterers of various permittivities. We also analyze two scattering problems involving semiconductors with high permittivity. Some concluding remarks are given in Section 4.

\section{Theory}

A. Integral Equation for the Coupled-Dipole Approximation

In this paper we consider a scatterer of relative permittivity $\varepsilon(\mathbf{r})$ embedded inside a two-layer background, as shown in Fig. 1. The structure is illuminated by an incident electric field $\mathbf{E}^{0}(\mathbf{r})$. We consider nonmagnetic materials $\left(\mu=\mu_{0}\right)$ and assume an $\exp (-i \omega t)$ time dependence.

The field $\mathbf{E}(\mathbf{r})$ scattered by this system is given by the integral equation

$$
\mathbf{E}(\mathbf{r})=\mathbf{E}^{0}(\mathbf{r})+\int_{V} \mathrm{~d} \mathbf{r}^{\prime} \mathbf{G}^{B}\left(\mathbf{r}, \mathbf{r}^{\prime}\right) \cdot k_{0}{ }^{2} \Delta \varepsilon\left(\mathbf{r}^{\prime}\right) \mathbf{E}\left(\mathbf{r}^{\prime}\right),
$$




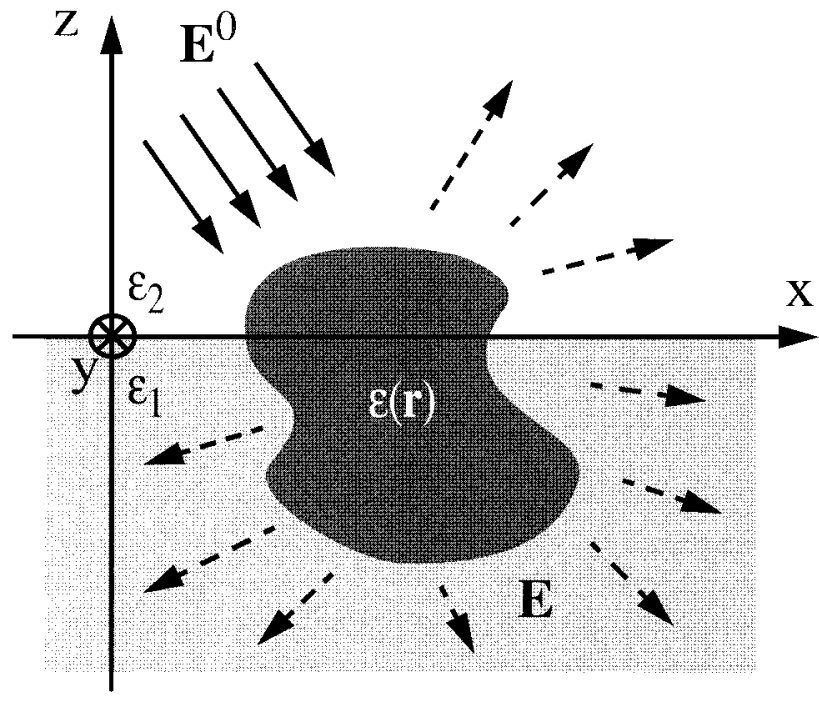

Fig. 1. Typical scattering problem considered in this study: The scatterer $\varepsilon(\mathbf{r})$ is deposited on, or embedded inside, a surface of permittivity $\varepsilon_{1}$ and illuminated with an incident field $\mathbf{E}^{0}(\mathbf{r})$. The upper medium has a permittivity $\varepsilon_{2}$.

where $\Delta \varepsilon\left(\mathbf{r}^{\prime}\right)$ is the dielectric contrast between the scatterer and the surrounding medium, which is given by

$$
\Delta \varepsilon(\mathbf{r})=\varepsilon(\mathbf{r})-\varepsilon_{B} .
$$

The background permittivity $\varepsilon_{B}$ depends on the $z$ coordinate of the point $\mathbf{r}$ and is defined by (Fig. 1)

$$
\begin{aligned}
\varepsilon_{B} & =\varepsilon_{1} & & \text { for } z<0 \\
& =\varepsilon_{2} & & \text { for } z \geq 0 .
\end{aligned}
$$

In Eq. (1) the integration runs over the entire scatterer volume $V ; \mathbf{G}^{B}\left(\mathbf{r}, \mathbf{r}^{\prime}\right)$ is the dyadic Green's tensor of the reference medium, with prime and nonprime variables associated with the source and the observation point, respectively; $k_{0}$ is the wave number in vacuum.

To solve Eq. (1) numerically, the volume of the scatterer is discretized into $N$ cells. Introducing $V_{i}$ and $\mathbf{r}_{i}$ for the volume and the position of the center of cell $i$, respectively, we can define $\left.\mathbf{E}(\mathbf{r})\right|_{\mathbf{r} \in V_{i}} \approx \mathbf{E}_{i}=$ $\mathbf{E}\left(\mathbf{r}_{i}\right)$ and $\left.\Delta \varepsilon_{i}(\mathbf{r})\right|_{\mathbf{r} \in V_{i}} \approx \Delta \varepsilon_{i}=\Delta \varepsilon\left(\mathbf{r}_{i}\right)$. In this way Eq. (1) can be rewritten as a dense system of linear equations,

$$
\begin{aligned}
\mathbf{E}_{i}= & \mathbf{E}_{i}^{0}+\sum_{j=1, j \neq i}^{N} \mathbf{G}_{i, j}^{B} \cdot k_{0}^{2} \Delta \varepsilon_{j} \mathbf{E}_{j} V_{j}+\mathbf{M}_{i} \cdot k_{0}^{2} \Delta \varepsilon_{i} \mathbf{E}_{i} \\
& -\mathbf{L} \cdot \frac{\Delta \varepsilon_{i}}{\varepsilon_{B}} \mathbf{E}_{i}, \quad i=1, \ldots, N,
\end{aligned}
$$

with

$$
\mathbf{M}_{i}=\lim _{\delta V \rightarrow 0} \int_{V_{i}-\delta V} \mathrm{~d} \mathbf{r}^{\prime} \mathbf{G}^{B}\left(\mathbf{r}_{i}, \mathbf{r}^{\prime}\right) .
$$

In Eq. (4) $M_{i}$ is the self-induction term for which approximations can be found in the literature. ${ }^{2}$ The source dyadic $\mathbf{L}$ depends on the exclusion volume shape and is tabulated in Ref. 10.

Note that Eq. (4) forms a large system of algebraic equations with $3 N$ equations and $3 N$ unknowns from which the electric field inside the scatterer can be found. The field outside the scatterer can then simply be obtained from the field inside the object by means of discretizing Eq. (1) for any $\mathbf{r} \notin V^{\prime}$.

One of the powerful features associated with Eq. (1) is that it can be used for solving configurations that include scatterers embedded inside an inhomogeneous background by use of the appropriate Green's tensor corresponding to this background. In this manner only the scatterers, not the background, must be discretized. In Subsection 2.B we derive the Green's tensor for the two-layer system depicted in Fig. 1.

\section{B. Surface Green's Tensor}

References 11 and 12 give expressions for the Green's tensors associated with horizontal and vertical dipoles embedded inside a stratified medium. These Green's tensors are first derived in the spectral domain, where analytical expressions can be obtained. They are then transformed into the direct space domain via numerical Sommerfield integrals..$^{13,11}$

In the quasi-static case, which can be used when the scatterers are small, these Sommerfield integrals take a simple form and can be solved analytically. ${ }^{14}$

Considering the system depicted in Fig. 1, with $z$ being the normal to the surface $\varepsilon_{1}$, for $z \geq 0$ and $z^{\prime} \geq$ 0 (i.e., for the observation point and the source point in the upper medium), the nonretarded Green's tensor $* \mathbf{G}\left(\mathbf{r}, \mathbf{r}^{\prime}\right)$ has the following form ${ }^{14}$ :

$$
* \mathbf{G}\left(\mathbf{r}, \mathbf{r}^{\prime}\right)=* \mathbf{G}^{2}\left(\mathbf{r}, \mathbf{r}^{\prime}\right) \mp \frac{\varepsilon_{1}-\varepsilon_{2}}{\varepsilon_{1}+\varepsilon_{2}} * \mathbf{G}^{2}\left(\mathbf{r}, \mathbf{r}^{\prime \prime}\right),
$$

where ${ }^{*} \mathbf{G}^{2}\left(\mathbf{r}, \mathbf{r}^{\prime}\right)$ is the quasi-static Green's tensor for an infinite homogeneous three-dimensional (3D) medium of permittivity $\varepsilon_{2}$. Note that ${ }^{*} \mathbf{G}^{2}\left(\mathbf{r}, \mathbf{r}^{\prime \prime}\right)$ takes into account the effect of an image source located at $\mathbf{r}^{\prime \prime}$. In Eq. (6) the minus sign and the plus sign, respectively, refer to horizontal ( $x$ - and $y$-directed) and vertical ( $z$-directed) sources, respectively.

For $z<0$ and $z^{\prime}<0$ the surface Green's tensor is given by

$$
* \mathbf{G}\left(\mathbf{r}, \mathbf{r}^{\prime}\right)=* \mathbf{G}^{1}\left(\mathbf{r}, \mathbf{r}^{\prime}\right) \pm \frac{\varepsilon_{1}-\varepsilon_{2}}{\varepsilon_{1}+\varepsilon_{2}} * \mathbf{G}^{1}\left(\mathbf{r}, \mathbf{r}^{\prime \prime}\right),
$$

where ${ }^{*} \mathbf{G}^{1}\left(\mathbf{r}, \mathbf{r}^{\prime}\right)$ is the Green's tensor for an infinite homogeneous $3 \mathrm{D}$ medium of permittivity $\varepsilon_{1}$. In this case the plus sign and the minus sign are associated with horizontal and vertical sources, respectively.

For $z<0$ and $z^{\prime} \geq 0$, the surface Green's tensor becomes

$$
* \mathbf{G}\left(\mathbf{r}, \mathbf{r}^{\prime}\right)=\frac{2 \varepsilon_{1}}{\varepsilon_{1}+\varepsilon_{2}} * \mathbf{G}^{1}\left(\mathbf{r}, \mathbf{r}^{\prime}\right),
$$


and for $z \geq 0$ and $z^{\prime}<0$ it is

$$
* \mathbf{G}\left(\mathbf{r}, \mathbf{r}^{\prime}\right)=\frac{2 \varepsilon_{2}}{\varepsilon_{1}+\varepsilon_{2}} * \mathbf{G}^{2}\left(\mathbf{r}, \mathbf{r}^{\prime}\right) .
$$

Equations (6)-(9) are valid for the regular Green's tensor and for the filtered Green's tensor. In the following, an expression associated with the filtered Green's tensor is derived in detail.

\section{Three-Dimensional Filtered Green's Tensor}

We derive the components of the 3D filtered Green's tensor by following the procedure described in Ref. 9 . The main idea is to filter out the components of the Green's tensor that limit the accuracy of the field representation in the discretized system.

\section{Fourier Transform}

The filtering procedure is performed in Fourier space with the 3D Fourier-transform pair defined in Ref. 9. Since the spatial function $g(x, y, z)$ is the scalar Green's function associated with an infinite homogeneous medium, it is only a function of the relative distance $R=\left|\mathbf{r}-\mathbf{r}^{\prime}\right|$ between source and observation points. Introducing spherical coordinates and performing angle integrations, we can write the pair Fourier transform in the simple form

$$
\begin{aligned}
& \tilde{g}(k)=4 \pi \int_{0}^{\infty} \mathrm{d} R g(R) j_{0}(k R) R^{2}, \\
& g(R)=\frac{1}{2 \pi^{2}} \int_{0}^{\infty} \mathrm{d} k \tilde{g}(k) j_{0}(k R) k^{2} .
\end{aligned}
$$

In Eqs. (10) and (11), $j_{0}(k R)$ is the spherical Bessel function defined with $j_{0}(k R)=\sin (k R) / k R$.

\section{Dynamic Green's Tensor}

The Green's tensor $\mathbf{G}^{B}\left(\mathbf{r}, \mathbf{r}^{\prime}\right)$ for an infinite homogeneous $3 \mathrm{D}$ background medium $\varepsilon_{B}$ is readily given by ${ }^{15}$

$$
\begin{aligned}
\mathbf{G}^{B}\left(\mathbf{r}, \mathbf{r}^{\prime}\right)= & \left(\mathbf{1}+\frac{i k_{B} R-1}{k_{B}^{2} R^{2}} \mathbf{1}\right. \\
& \left.+\frac{3-3 i k_{B} R-k_{B}^{2} R^{2}}{k_{B}^{2} R^{4}} \mathbf{R R}\right) \frac{\exp \left(i k_{B} R\right)}{4 \pi R} .
\end{aligned}
$$

In Eq. (12), 1 is the unit Green's tensor, $R=|\mathbf{R}|=\mid \mathbf{r}-$ $\mathbf{r}^{\prime} \mid$ is the relative distance between source and observation points, and $k_{B}$ is the wave number of the background medium. We can derive the filtered Green's tensor for an infinite homogeneous 3D background medium $\mathbf{G}^{F}\left(\mathbf{r}, \mathbf{r}^{\prime}\right)$ in a similar way, looking for the filtered scalar Green's function $g^{F}\left(\mathbf{r}, \mathbf{r}^{\prime}\right)$ and then deriving the Green's tensor with the following relation:

$$
\mathbf{G}^{F}\left(\mathbf{r}, \mathbf{r}^{\prime}\right)=\left(1+\frac{\boldsymbol{\nabla} \nabla}{k_{B}^{2}}\right) g^{F}\left(\mathbf{r}, \mathbf{r}^{\prime}\right) .
$$

We find the Fourier transform of $g^{B}\left(\mathbf{r}, \mathbf{r}^{\prime}\right)$ by introducing $g(R)=\exp \left(i k_{B} R\right) / 4 \pi R$ into Eq. (10). We obtain

$$
\tilde{g}(k)=\frac{1}{k^{2}-k_{B}^{2}} .
$$

Then the inverse Fourier transform must be applied to Eq. (14) by use of Eq. (11), where the integration region must be limited to the passthrough region of the filter, i.e., to $k=0, \ldots, k_{F}$. Since the function $\tilde{g}(k)$, as defined by Eq. (14), has a pole within the passthrough region, for $k=k_{\rho}$, we instead use the complementary filter, integrating for $k=k_{F}, \ldots, \infty$, and subtract the result from the nonfiltered function $^{9}$ :

$$
g^{F}(R)=g(R)-\frac{1}{2 \pi^{2}} \int_{k_{F}}^{\infty} \mathrm{d} k \tilde{g}(k) j_{0}(k R) k^{2} .
$$

Introducing Eq. (14) into Eq. (15), we find for the filtered scalar Green's function

$$
\begin{aligned}
g^{F}(R)= & \frac{\exp \left(j k_{B} R\right)}{4 \pi R}-\frac{\sin \left(k_{B} R\right)}{4 \pi^{2} R}\left\{\operatorname{Ci}\left[\left(k_{F}+k_{B}\right) R\right]\right. \\
& \left.-\operatorname{Ci}\left[\left(k_{F}-k_{B}\right) R\right]\right\}-\frac{\cos \left(k_{B} R\right)}{4 \pi^{2} R} \\
& \times\left\{\pi-\operatorname{Si}\left[\left(k_{F}+k_{B}\right) R\right]-\operatorname{Si}\left[\left(k_{F}-k_{B}\right) R\right]\right\} .
\end{aligned}
$$

The filtered Green's tensor can then be derived from Eq. (13); it has the form

$$
\begin{aligned}
\mathbf{G}^{F}\left(\mathbf{r}, \mathbf{r}^{\prime}\right)= & \left(g^{F}+\frac{g^{\prime F}}{k_{B}^{2} R}\right) \mathbf{1}+\left(\frac{-g^{\prime F}}{k_{B}^{2} R^{3}}+\frac{g^{\prime \prime F}}{k_{B}^{2} R^{2}}\right) \mathbf{R} \mathbf{R} \\
& +\frac{\mathbf{1}}{3 k_{\mathrm{ref}}^{2}} h^{r}\left(\mathbf{r}-\mathbf{r}_{k}\right) .
\end{aligned}
$$

All the terms included in this equation are given in Appendix A.

\section{Quasi-Static Green's Tensor}

For the quasi-static case the Green's tensor associated with an infinite homogeneous 3D background medium is obtained from Eq. (12) with $k_{B} \rightarrow 0$. It is given by

$$
\mathbf{G}_{\mathrm{qs}}^{B}\left(\mathbf{r}, \mathbf{r}^{\prime}\right)=\left(\frac{-1}{k_{B}^{2} R^{2}} \mathbf{1}+\frac{3}{k_{B}^{2} R^{4}} \mathbf{R} \mathbf{R}\right) \frac{1}{4 \pi R} .
$$

To obtain the filtered Green's tensor, we operate in a similar way as in the dynamic case: A Fourier transform is applied to the scalar Green's function associated with the background for $k_{B} \rightarrow 0$. This leads to

$$
\tilde{g}(k)=\frac{1}{k^{2}} \text {. }
$$




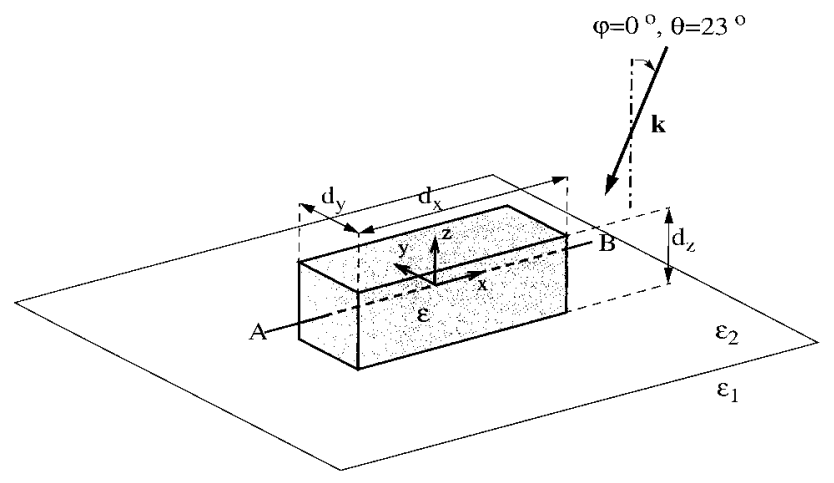

Fig. 2. Geometry used for the calculations: A rectangular scatterer with dimensions $d_{x}=110 \mathrm{~nm}, d_{y}=55 \mathrm{~nm}, d_{z}=55 \mathrm{~nm}$ is deposited on a surface. The scatterer and the surface have the same permittivity (see text). This system is illuminated with a plane wave propagating at $\theta=23^{\circ}$. The incident electric field $\mathbf{E}^{0}$ is linearly polarized, with a $30^{\circ}$ angle from the $p$-polarization axis.

Introducing Eq. (19) into Eq. (15) where $g(R)=$ $1 / 4 \pi R$, the following equation is obtained,

$$
g_{\mathrm{qs}}^{F}=\frac{1}{4 \pi R}-\frac{1}{4 \pi^{2} R}\left[\pi-2 \operatorname{Si}\left(k_{F} R\right)\right] .
$$

The filtered Green's tensor has then the form,

$$
\begin{aligned}
\mathbf{G}_{\mathrm{qs}}^{F}\left(\mathbf{r}, \mathbf{r}^{\prime}\right)= & \left(\frac{g_{\mathrm{qs}}^{\prime F}}{k_{B}^{2} R}\right) 1+\left(\frac{-g_{\mathrm{qs}}^{\prime F}}{k_{B}^{2} R^{3}}+\frac{g_{\mathrm{qs}}^{\prime \prime F}}{k_{B}^{2} R^{2}}\right) \mathbf{R R} \\
& +\frac{1}{3 k_{\mathrm{ref}}^{2}} h^{r}\left(\mathbf{r}-\mathbf{r}_{k}\right) .
\end{aligned}
$$

All the terms included in this equation are also given Appendix A.

\section{Results and Discussion}

In this section we first study the limitation of regular CDA for large-permittivity scatterers on a surface

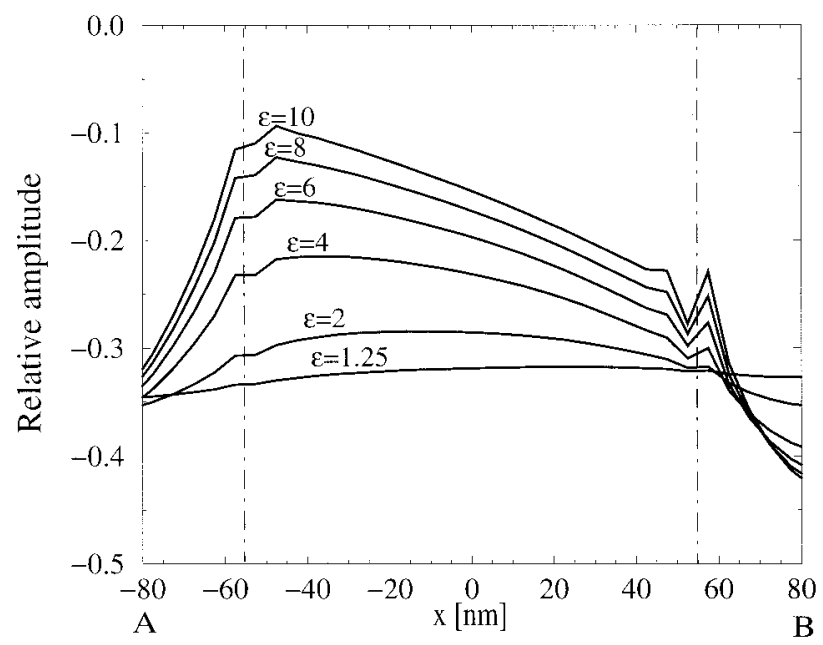

Fig. 3. Amplitude of the $E_{z}$ field component at the center of the structure depicted in Fig. 2 (the field is calculated along the $A B$ line shown in Fig. 2). The vertical dotted-dashed lines represent the scatterer boundary. Nonfiltered Green's tensor.
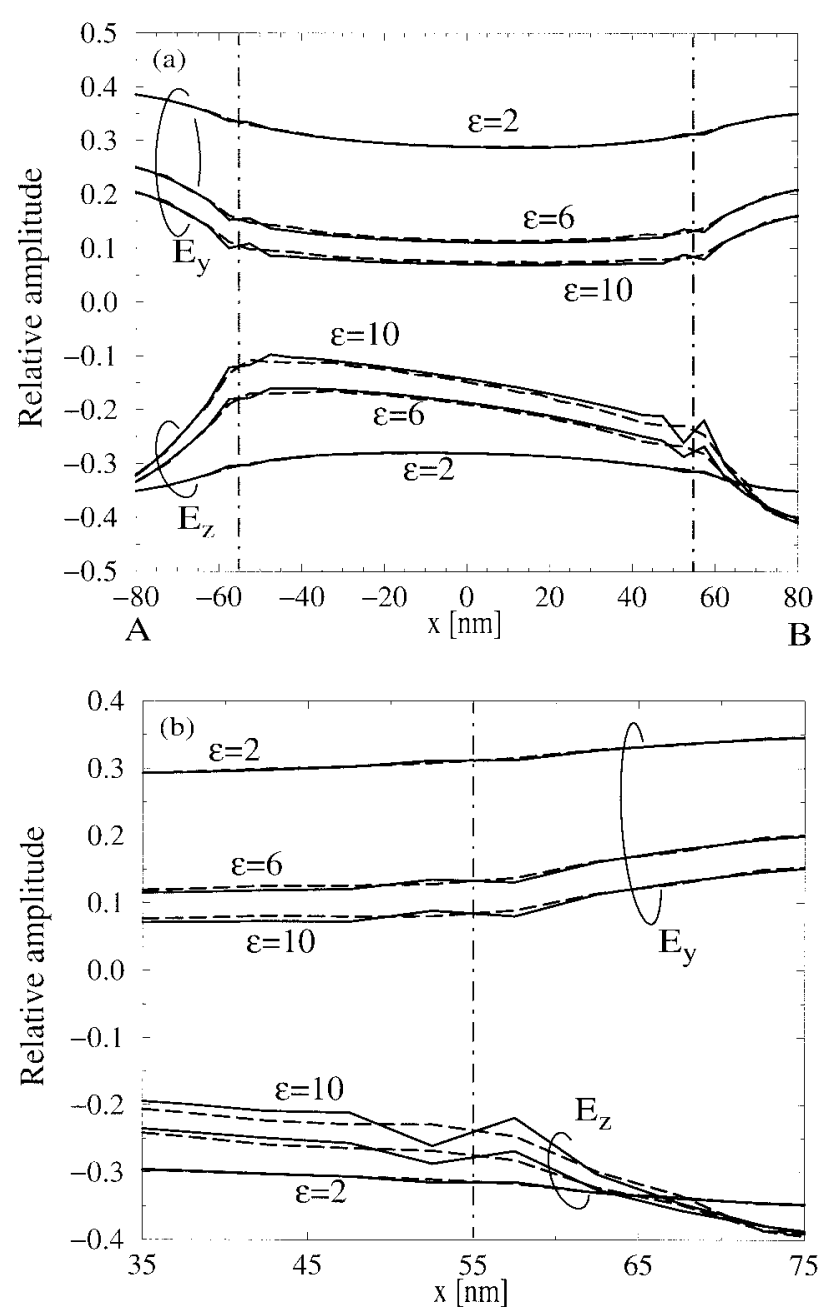

Fig. 4. Comparison between nonfiltered (continuous curve) and filtered (dashed curve) solutions for the two continuous field components $E_{y}$ and $E_{z}$. (a) Same region as in Fig. 3. (b) Blowup of the region surrounding the right-hand interface.

and show how these limitations can be overcome by use of the filtered CDA developed in Section 2. We then apply our new scheme to a practical example produced by semiconductor technology.

\section{A. Assessment of the Method}

Let us consider a scatterer with moderate dimensions so that the quasi-static model-which is the core of this study - can be used. ${ }^{14}$ Figure 2 depicts the geometry under study: A small homogeneous scatterer with permittivity $\varepsilon$ is deposited on a surface with the same permittivity $\left(\varepsilon_{1}=\varepsilon\right)$. We first consider a scatterer with the following dimensions: $d_{x}=110 \mathrm{~nm}, d_{y}=55 \mathrm{~nm}, d_{z}=55 \mathrm{~nm}$; it is discretized with a $\Delta_{x}=\Delta_{y}=\Delta_{z}=5 \mathrm{~nm}$ mesh. This system is illuminated with a plane wave propagating at $\theta=$ $23^{\circ}$, linearly polarized, with a $30^{\circ}$ polarization angle from the $p$-polarization axis. The illumination wavelength in vacuum is $\lambda=1000 \mathrm{~nm}$.

In the following we vary the permittivity $\varepsilon$ of the scatterer and the surface and study its influence on the result accuracy. To measure this accuracy, we 


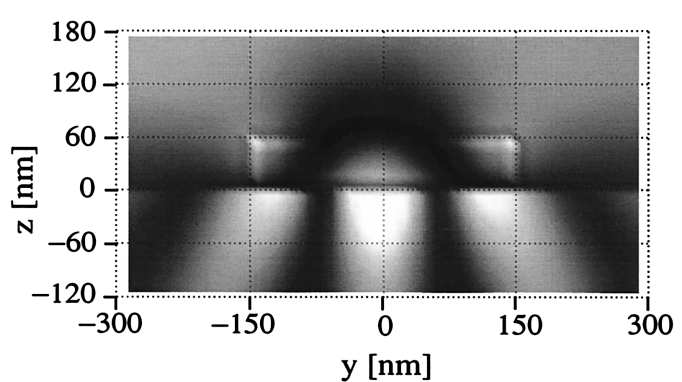

(a)

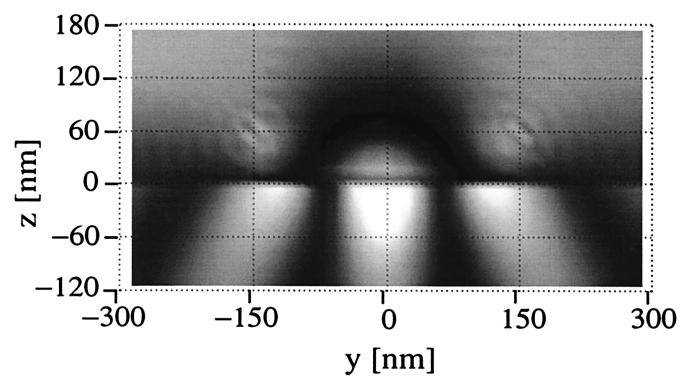

(b)

Fig. 5. Intensity $E_{x} E_{x}^{*}$ of the $x$ electric field component along the center of a scatterer with dimensions $d_{x}=300 \mathrm{~nm}, d_{y}=300 \mathrm{~nm}$, $d_{z}=60 \mathrm{~nm}$ and permittivity $\varepsilon=10$. The substrate has $\varepsilon_{1}=10$, and the illumination wavelength in $\lambda=600 \mathrm{~nm}$. (a) Nonfiltered Green's tensor, (b) filtered Green's tensor.

consider how well the boundary conditions required by Maxwell's equations are fulfilled by our numerical results.

In Fig. 3 we show the amplitude $E_{z}$ of the $z$ component of the electric field computed with the regular Green's tensor along the $A B$ line in the middle of the structure (Fig. 2), for six different scatterer permittivities $\varepsilon$.

This field component should be continuous upon

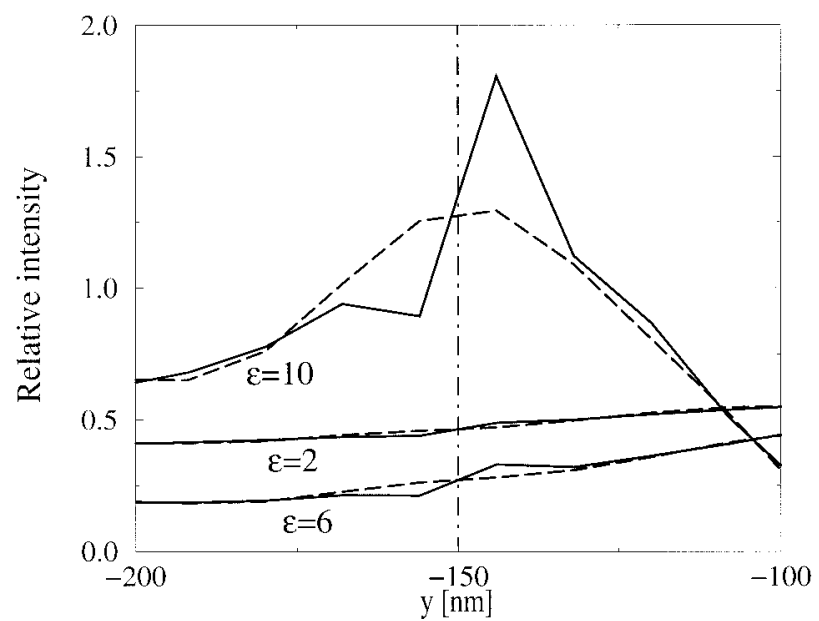

Fig. 6. Field continuity at the left-hand interface of the scatterer depicted in Fig. 5 for three different permittivities $\varepsilon=\varepsilon_{1}=2,6$, or $10 ; \lambda=600 \mathrm{~nm}$. The intensity of the $x$ electric field component is shown for the nonfiltered (continuous curve) and the filtered (dashed curve) Green's tensor.
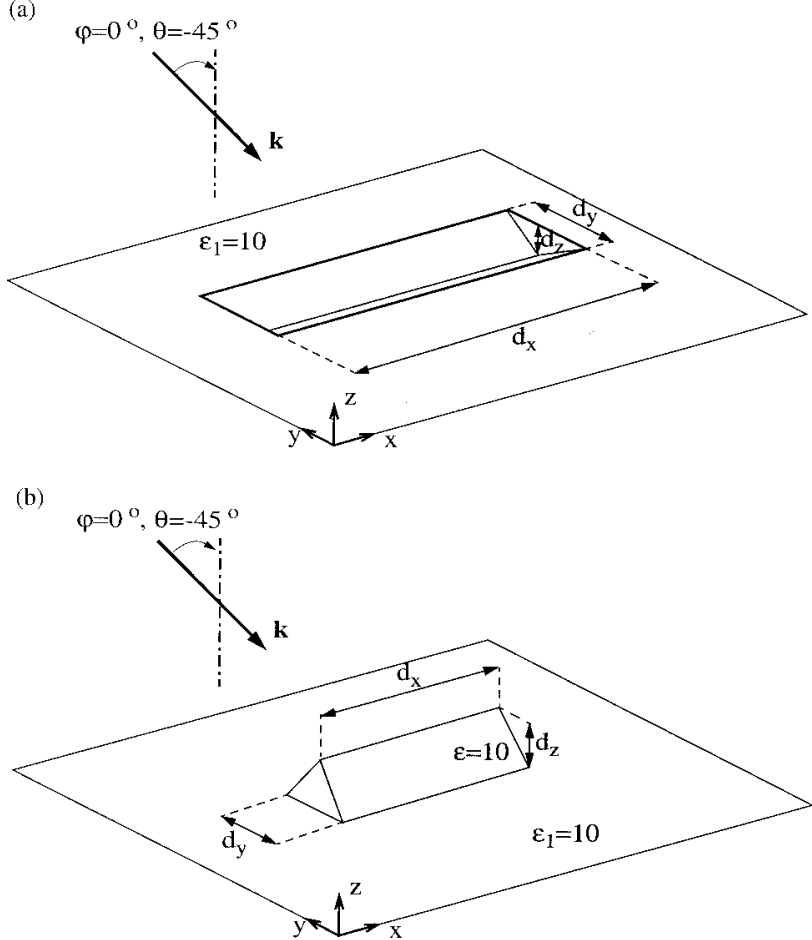

Fig. 7. Simulation of defects on a high-permittivity substrate $\left(\varepsilon_{1}=10\right)$. (a) Trench $\left(d_{x}=600 \mathrm{~nm}, d_{y}=100 \mathrm{~nm}, d_{z}=50 \mathrm{~nm}\right)$ is etched in the substrate. (b) Mesoscopic protrusion $\left(d_{x}=600 \mathrm{~nm}\right.$, $d_{y}=100 \mathrm{~nm}, d_{z}=50 \mathrm{~nm}$ ) with similar permittivity is deposited on the surface.

crossing the air-scatterer interfaces at $x= \pm 55 \mathrm{~nm}$, because it is parallel to the material interfaces. ${ }^{16}$ In Fig. 3 we see that with the regular Green's tensor this boundary condition is fulfilled only for scatterers with low permittivity. Even for $\varepsilon=2$ the field is discontinuous at the material interfaces. This effect increases for larger permittivities.

In Fig. 4(a) we give similar results for the selected permittivities $\varepsilon=2,6$, and 10 . Both $E_{y}$ and $E_{z}$ are represented. These two field components should be continuous. In addition to the regular Green's tensor, we now also show the results obtained with the filtered Green's tensor described in Subsection 2.C.3. The field obtained with this new scheme now perfectly fulfills the boundary conditions, even for a permittivity as high as $\varepsilon=10$. This is also visible in Fig. 4(b), where we present a blowup of the region surrounding the right-hand material interface. Note how well the boundary conditions are fulfilled with the filtered scheme.

In Ref. 14 we showed that the quasi-static approximation can be successfully used to investigate scatterers of larger dimensions, typically as great as 1 wavelength. In Fig. 5 we consider a highpermittivity scatterer $(\varepsilon=10)$ with dimensions $d_{x}=$ $d_{y}=300 \mathrm{~nm}, d_{z}=60 \mathrm{~nm}$ (see Fig. 2), located on a surface with a similar permittivity. The illumination conditions are similar to those in the previous example, now with a wavelength of $\lambda=600 \mathrm{~nm}$. Figure 5 shows the intensity of the $x$ electric field 
(a)

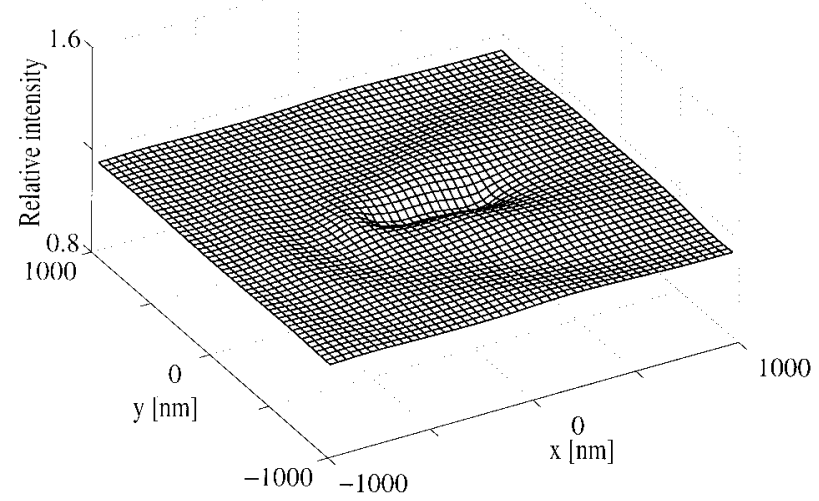

(b)

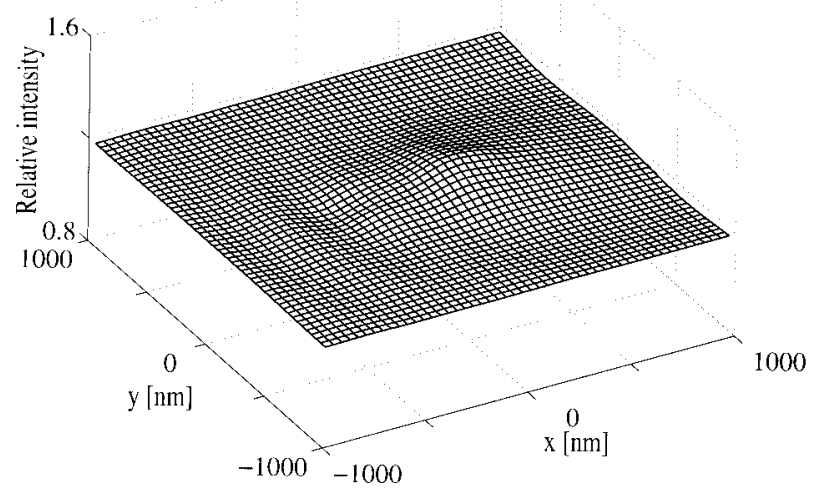

Fig. 8. Relative field intensity at a constant height $x=100 \mathrm{~nm}$ above the substrate, when a trench is etched in the dielectric [geometry of Fig. 7(a)]. Two incident polarizations are investigated: (a) $p$ polarization and (b) $s$ polarization. The wavelength is $\lambda=633 \mathrm{~nm}$.

component computed with the regular and the filtered Green's tensors, in the plane $x=0$. This field component should be continuous across all the different material interfaces. We see in Fig. 5(a) that this is not the case when the field is computed with the regular Green's tensor: Depolarization fields arise at the material interfaces, disrupting the continuity of this field component and rendering the scatterer boundary clearly visible. This is particularly the case at the proximity of the scatterers' corners at $z=60 \mathrm{~nm}$ and $y= \pm 150 \mathrm{~nm}$. However, when the filtered Green's tensor is used, the field is perfectly continuous, and the object boundary is invisible [Fig. 5(b)].

This effect is investigated in more detail in Fig. 6, where we show the intensity of the $x$ field component at the vicinity of the left-hand-side vertical interface, evaluated along a line defined by $x=0 \mathrm{~nm}, z=30$ $\mathrm{nm}$. Different scatterers and surface permittivities $\varepsilon$ are considered. Note the discrepancy between the regular and the filtered solutions, especially for highpermittivity scatterers (Fig. 6). Striking in Fig. 6 is how well the field continuity is fulfilled with the filtered scheme, even for $\varepsilon=10$.

These results give us some assurance that the fil-

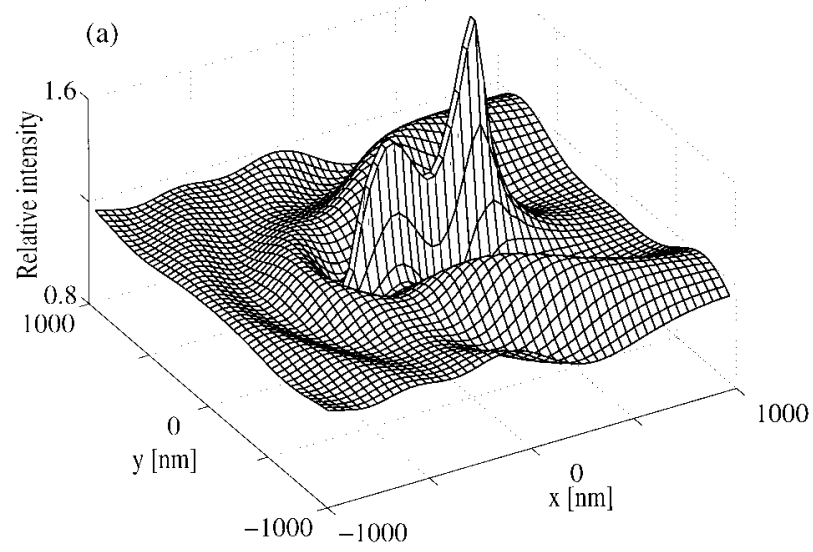

(b)

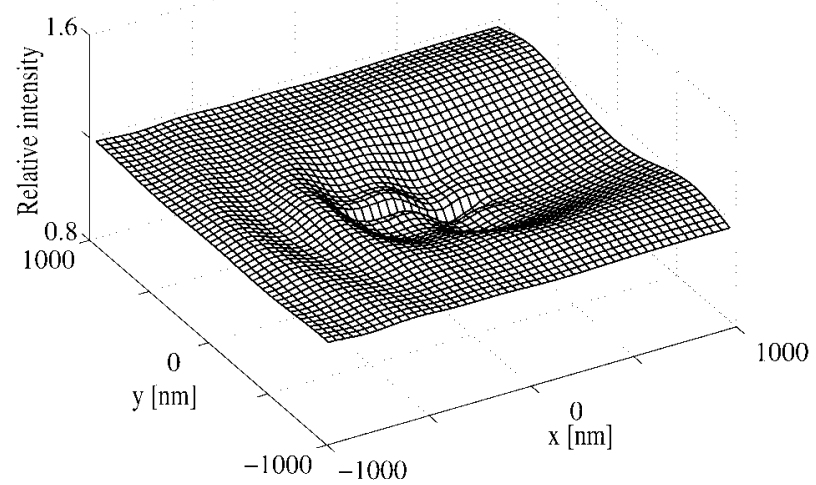

Fig. 9. Relative field intensity at a constant height $x=100 \mathrm{~nm}$ above the substrate, when a protrusion is deposited on the surface [geometry of Fig. 7(b)]. Two incident polarizations are investigated: (a) $p$ polarization and (b) $s$ polarization. The wavelength is $\lambda=633 \mathrm{~nm}$.

tered Green's tensor developed in Section 2 can be used to investigate high-permittivity scatterers accurately.

\section{B. Defects on a Semiconductor Wafer}

In this section we illustrate the application of the new filtered scheme to a practical problem.

Optical wafer inspection is widely used in semiconductor manufacturing plants to locate defects on a semiconductor wafer. ${ }^{17}$ At optical frequencies most semiconductors have a high permittivity, of the order of $\varepsilon=10$. Our computation technique is therefore well suited for investigating such wafer-inspection techniques and computing the field scattered by small defects on a semiconductor substrate.

To illustrate this, we investigate the two geometries depicted in Fig. 7: a scratch in a highpermittivity surface and a defect on that surface.

Figure 8 shows the relative total field intensity in a plane parallel to the surface $(z=100 \mathrm{~nm})$ for the geometry of Fig. 7(a) (scratch in the surface). For $p$ polarization the field distribution reproduces the defect shape, with a depletion in the field-intensity distribution just above the scratch. In addition, a small 
interference pattern resulting from the interaction of the illuminating field with the scattered one is visible. A contrast reversal is observed for the other illumination polarization [Fig. 8(b)]. This type of reversal is well known from near-field optics. ${ }^{18}$

The defect above the surface shown in Fig. 9 gives a stronger signal than the scratch. With the backscattered field being stronger, so is the interference pattern. A similar contrast reversal, with the surface defect appearing now as a depletion in the field distribution, is observed when the illumination polarization is changed [Fig. 9(b)].

\section{Conclusions}

In this paper we have proposed a filtered nonretarded scheme capable of analyzing small scatterers of high permittivity embedded inside or deposited above a surface. We have formally deduced the filtered Green's tensor associated with a two-layer medium in the quasi-static approximation. We have shown that the filtered scheme provides much more accurate results, allowing for the simulation of highpermittivity scatterers. In particular, the results obtained with this new scheme more accurately fulfill the boundary conditions imposed by Maxwell's equations.

This new technique should be useful for the scattering calculations of small defects on highpermittivity surfaces, such as semiconductors. We shall soon provide public access to the code for computing the filtered Green's tensor at the Computer Physics Communications Program Library. ${ }^{19}$

\section{Appendix A: Expressions for the Filtered Green's Tensor}

The following terms correspond to Eq. (17):

$$
\begin{aligned}
h^{r}(\mathbf{r})= & \frac{\sin \left(k_{F}|\mathbf{r}|\right)-k_{F}|\mathbf{r}| \cos \left(k_{F}|\mathbf{r}|\right)}{2 \pi|\mathbf{r}|^{3}} \\
g^{F}= & g^{B}-\frac{\alpha}{4 \pi^{2} R}, \\
g^{\prime F}= & i k_{B} g^{B}-\frac{g^{B}}{R}+\frac{\alpha}{4 \pi^{2} R^{2}}-\frac{\alpha^{\prime}}{4 \pi^{2} R}, \\
g^{\prime \prime F}= & -k_{B}^{2} g^{B}-\frac{2 i k_{B} g^{B}}{R}+\frac{2 g^{B}}{R^{2}}-\frac{\alpha}{2 \pi^{2} R^{3}}+\frac{\alpha^{\prime}}{2 \pi^{2} R^{2}} \\
& -\frac{\alpha^{\prime \prime}}{4 \pi^{2} R},
\end{aligned}
$$

where

$$
\begin{aligned}
\alpha= & \sin \left(k_{B} R\right)\left[\mathrm{Ci}^{+}-\mathrm{Ci}^{-}\right]+\cos \left(k_{B} R\right)\left[\pi-\mathrm{Si}^{+}-\mathrm{Si}^{-}\right], \\
\alpha^{\prime}= & k_{B} \sin \left(k_{B} R\right)\left[-\pi+\mathrm{Si}^{-}+\mathrm{Si}^{+}\right]+k_{B} \cos \left(k_{B} R\right) \\
& \times\left[\mathrm{Ci}^{+}-\mathrm{Ci}^{-}\right]-\frac{2 \sin \left(k_{F} R\right)}{R},
\end{aligned}
$$

$$
\begin{aligned}
\alpha^{\prime \prime}= & k_{B}^{2} \sin \left(k_{B} R\right)\left[\mathrm{Ci}^{-}-\mathrm{Ci}^{+}\right]+k_{B}^{2} \cos \left(k_{B} R\right)\left[\pi-\mathrm{Si}^{-}\right. \\
& \left.+\mathrm{Si}^{+}\right]+\frac{2 \sin \left(k_{F} R\right)}{R^{2}}-\frac{2 k_{F} \cos \left(k_{F} R\right)}{R},
\end{aligned}
$$

with $\mathrm{Ci}^{ \pm}=\mathrm{Ci}\left[\left(k_{F} \pm k_{B}\right) R\right]$ and $\mathrm{Si}^{ \pm}=\operatorname{Si}\left[\left(k_{F} \pm k_{B}\right) R\right]$.

The following terms correspond to Eq. (21):

$$
\begin{aligned}
& g_{\mathrm{qs}}^{F}=g_{\mathrm{qs}}^{B}-\frac{\alpha_{\mathrm{qs}}}{4 \pi^{2} R}, \\
& g_{\mathrm{qs}}^{\prime F}=-\frac{g_{\mathrm{qs}}^{B}}{R}+\frac{\alpha_{\mathrm{qs}}}{4 \pi^{2} R^{2}}-\frac{\alpha_{\mathrm{qs}}^{\prime}}{4 \pi^{2} R}, \\
& g_{\mathrm{qs}}^{\prime \prime F}=\frac{2 g_{\mathrm{qs}}^{B}}{R^{2}}-\frac{\alpha_{\mathrm{qs}}}{2 \pi^{2} R^{3}}+\frac{\alpha_{\mathrm{qs}}^{\prime}}{2 \pi^{2} R^{2}}-\frac{\alpha_{\mathrm{qs}}^{\prime \prime}}{4 \pi^{2} R},
\end{aligned}
$$

where

$$
\begin{aligned}
& \alpha_{\mathrm{qs}}=\pi-2 \operatorname{Si}\left(k_{F} R\right), \\
& \alpha_{\mathrm{qs}}^{\prime}=-\frac{2 \sin \left(k_{F} R\right)}{R}, \\
& \alpha_{\mathrm{qs}}^{\prime \prime}=\frac{2 \sin \left(k_{F} R\right)}{R^{2}}-\frac{2 k_{F} \cos \left(k_{F} R\right)}{R} .
\end{aligned}
$$

This research was supported by the Swiss National Science Foundation.

\section{References and Note}

1. E. M. Purcell and C. R. Pennypacker, "Scattering and absorption of light by nonspherical dielectric grains," Astrophys. J. 186, 705-714 (1973).

2. G. H. Goedecke and S. G. O'Brien, "Scattering by irregular inhomogeneous particles via the digitized Green's function algorithm," Appl. Opt. 27, 2431-2438 (1988).

3. W. T. Doyle, "Optical properties of a suspension of metal spheres," Phys. Rev. B 39, 9852-9858 (1989).

4. O. J. F. Martin, "3D Simulations of the experimental signal measured in near-field optical microscopy," J. Microsc. (Oxford) 194, 235-239 (1999).

5. H. Schmid, H. Biebuyck, B. Michel, and O. J. F. Martin, "Lightcoupling masks for lensless, sub-wavelength optical lithography," Appl. Phys. Lett. 72, 2379-2381 (1998).

6. M. Paulus, P. Gay-Balmaz, and O. J. F. Martin, "Accurate and efficient computation of the Green's tensor for stratified media," Phys. Rev. E 62, 5797-5807 (2000).

7. M. Paulus and O. J. F. Martin, "Light propagation and scattering in stratified media: a Green's tensor approach," J. Opt. Soc. Am. A 18, 854-861 (2001).

8. N. B. Piller and O. J. F. Martin, "Extension of the generalized multipole technique to anisotropic media," Opt. Commun. 150, 1-6 (1998).

9. N. B. Piller and O. J. F. Martin, "Increasing the performances of the coupled-dipole approximation: a spectral approach," IEEE Trans. Antennas Propag. 46, 1126-1137 (1998).

10. A. D. Yaghjian, "Electric dyadic Green's functions in the source region," Proc. IEEE 68, 248-263 (1980).

11. J. R. Mosig, "Integral equation technique," in Numerical Techniques for Microwave and Millimeter-Wave Passive Structures, T. Itoh, ed. (Wiley, New York, 1989), Chap. 3, pp. 133-213.

12. P. Gay-Balmaz and J. R. Mosig, "3D planar radiating structures in stratified media," Int. J. Microwave Millimeter Wave CAE 3, 330-343 (1997). 
13. L. B. Felsen and N. Marcuvitz, Radiation and Scattering of Waves (Institute of Electrical and Electronics Engineers, New York, 1994).

14. P. Gay-Balmaz and O. J. F. Martin, "Validity domain and limitation of non-retarded Green's tensor for electromagnetic scattering at surfaces," Opt. Commun. 184, 37-47 (2000).

15. H. Levine and J. Schwinger, "On the theory of electromagnetic wave diffraction by an aperture in an infinite plane conducting screen," Comments Pure Appl. Math. 3, 355-391 (1950).
16. J. D. Jackson, Classical Electrodynamics, 3rd ed. (Wiley, New York, 1999).

17. See, for example, the International Technology Roadmap for Semiconductors, http://public.itrs.net.

18. O. J. F. Martin, C. Girard, and A. Dereux, "Dielectric versus topographic contrast in near-field microscopy," J. Opt. Soc. Am. A 13, 1801-1808 (1996).

19. The Computer Physics Communications Program Library, http://www.cpc.cs.qub.ac.uk. 